\title{
GROUPAGE CARGO TRANSPORTATION MODEL
}

\author{
Ruslans Aleksejevs ${ }^{1}$, Raufs Guseinovs ${ }^{2}$, \\ Alexander N. Medvedev ${ }^{3}$, Sharif E. Guseynov ${ }^{4}$ \\ ${ }^{1}$ Riga State Gymnasium No. 1 \\ 8 Raina Boulevard, Riga LV-1050, Latvia \\ e-mail: ruslan.alekseyev.1998@gmail.com \\ ${ }^{2}$ Faculty of Humanities, Leiden University \\ Rapenburg 70, 2311 EZ Leiden, the Netherlands \\ e-mail: r.guseinovs@umail.leidenuniv.nl \\ ${ }^{3}$ Department of Aviation Transport, Transport and Telecommunication Institute \\ 1 Lomonosov Street, Riga LV-1019, Latvia \\ e-mail: medvedevs.a@tsi.lv \\ ${ }^{4}$ Institute of Mathematical Sciences and Information Technologies, Liepaja University \\ 14 Liela Street, Liepaja LV-3401, Latvia
}

\begin{abstract}
In this work we consider a specific problem of optimal planning of maritime transportation of multiproduct cargo by ships of one (corporate strategy) or several (partially corporate strategy) companies: the core of the problem consists of the existence of the network of intermediate seaports (i.e. transitional seaports), where for every ship arrived the cargo handling is done, and which are situated between the starting and the finishing seaports. In this work, there are mathematical models built from scratch in the form of multicriteria optimization problem; then the goal attainment method of Gembicki is used for reducing the built models to a onecriterion problem of linear programming.
\end{abstract}

Keywords: Marine cargo transportation, mathematical modelling, multicriteria optimization, Pareto efficient solution, goal attainment method, regularized solution

\section{Introduction}

Maritime cargo transportation is a complicated multistage transportation-and-manufacturing process, in what, besides sender, carrier and recipient of cargo, other natural and legal persons do also participate (Nikolaeva and Tsymbal, 2005; James and Kendall, 2008): agents and forwarders; banks and insurance companies; seaport workers and workers of logistic complexes of intermodal and multimodal transportation systems; representatives of state supervisory authorities; etc. All these transportation-andmanufacturing process participants at different stages of cargo transportation enter into specific legal relationships among themselves for solving particular problems, which assists with the achievement of the common objective - punctual (fastness), inexpensive (economic expedience) and secure (safety and stability) delivery of the cargo to the destination. For instance, the article (Abusdal, 2012) considers various optimization models solving the fleet size decision making problems involving fleet changes during several planning periods. In the article the choice of the best suited model structure of deterministic nature is described. Further, the article (Liotta et al., 2015) suggests a model integrating supply, production networks and sustainable freight transportation for strategic and tactical decision making. The objective function considers sourcing, production and transportation costs as well as carbon dioxide emissions as environmental impacts of transport over a multimodal network. The work (Kang et al., 2012) in detail expounds a metaheuristic algorithm based on a genetic algorithm. The purpose of the developed algorithm consists in solving the problem of car carriers' work's efficiency as well as in creating a maritime transportation planning support system, thus making it possible to prepare various alternatives, evaluate them and, consequently, support user's decision making.

The maritime cargo transportation process itself can be considered as a material commodity flow, whose necessity, direction and properties are generally determined by the needs of international trade. The motion of this flow is realized by the hardware of the merchant marine and seaports in the presence of proper technologies and scientifically substantiated strategy, which are necessary at every stage of the transportation for ensuring fastness, economic expedience and safety of transportation of the cargo to the destination (Nikolaeva and Tsymbal, 2005; Song and Panayides, 2012). In whole, the process of maritime 
cargo transportation must be accompanied and supported by full and reliable dataware and other important kinds of support: technical, technological, organizational, commercial, financial and legal supports.

Merchant marine as an economic sector not only experiences the influence of the processes occurring here, but also actively influences the world economy itself (Masane-Ose, 2014; Nikolaeva and Tsymbal, 2005; Wakeman and Bomba, 2010). Under present-day conditions of globalization and intensively developing internationalization of manufacturing stable, uninterrupted transport maintenance of the international commodity exchange is becoming an indispensable condition of normal functioning and development of particular national economies as well as the world economy on the whole. Currently, the development of international navigation and the processes of the world economic system have a close link, an interrelationship: abnormality in maritime cargo transportation can have a destructive effect on the world economy.

For the last 20 years the portion of transportation expenses in the international price of a good has fallen, on average, $10 \%$, but the cost of the transported goods has spiked up, which led to increase of insurance premiums: in 2014, in comparison with 2000, they increased 1.2 times (in comparison with 1980 - 7 times), and according to predictions the growth trend will be stable at least until 2025 (Swiss Re Economic Research \& Consulting, 2001-2015). That is why in these conditions qualitative factors of transportation service (speed; cost; safety and stability; service), which define the level of competitive ability of national transport systems, in particular, the level of maritime cargo transportation, is becoming priority-driven. Some EU countries like the Baltic States (Latvia, Lithuania, and Estonia), the Netherlands, Germany, Hungary, Czech Republic, and Poland, owning small territories, use their advantageous geographical locations to turn transit into significant income items for their national budgets (Masane-Ose, 2014). For instance, the Netherlands are the most important transit crossing of EU, and the portion of incomes from transit of freight flows going through the Port of Rotterdam form more than $45 \%$ of total volume of export of services of the Netherlands (World Port Source, 2015).

In this work we investigate a specific problem, which is largely typical exactly for the seaports. The problem heart consists in the following: it is required to create a plan of maximal multiproduct cargo transportation by every ship through the given sea route such that the transportation expenses are minimal if (1) the cost of transportation of one unit of cargo between any two intermediate seaports by every ship is minimal; (2) the volumes of cargo unloaded and/or loaded in every intermediate seaport are known; (3) is known the volume of intended to be transported accumulated cargo in every intermediate seaport; (4) is known the shipload of every ship after the intended to be transported to this seaport cargo is unloaded from it. It is obvious that in the formulated problem every intermediate seaport is simultaneously a departure and destination point. In the work (Medvedeva, 2014) reasons of this specific cargo transportation are expanded, and is made comparative analysis in comparison with other sea routes in transpacific, transatlantic and Asia $\rightarrow$ Europe directions.

In the considered particular problem with respect to the ships performing transportations through the given sea route we can say the following: the ships can belong to one company, and, therefore, in this case we have a corporate strategy for performing a transportation; all or part of the ships can belong to different companies, and, therefore, in this case we can speak about a partially corporate strategy of performing a transportation. Obviously, in the case of partially corporate strategy it is possible to add to the formulation of the problem different conditions and constraints and, as result, obtain various problems according to their degree of complexity and purpose.

\section{Construction of quantitative model}

The problem described in the introduction, can be schematically presented in the form of the following directed graph:

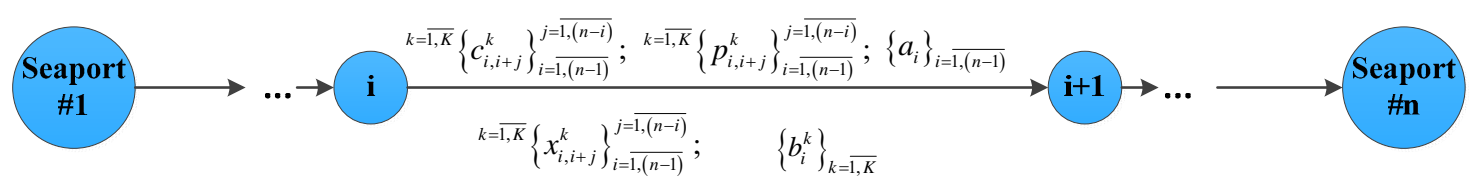

Figure 1. Graphic illustration of cargo transportation among $n$ seaports by $K$ ships. All the numerical parameters shown above and below the edges, connecting two adjacent seaports $i$ and $(i+1)$, characteristic also for the rest of the edges, except the last one: above the edge $(n-1, n)$ there is no parameter $p$, because for $\forall k=\overline{1, K}$ holds $p_{n-1, n}^{k} \equiv 100$. 
Before we begin the model building for the considered problem, let's introduce the following denotations: $n \in N$ denotes the amount of seaports between which the cargo transportation is performed; $\left[T_{\text {start }}, T_{\text {end }}\right]$ denotes a period of time during which the cargo transportation is performed; $K \in N$ denotes the total amount of ships used during the period of time $\left[T_{\text {start }}, T_{\text {end }}\right]$ for cargo transportation along the route shown on Fig. $1 ; R_{++}^{m} \stackrel{\text { def }}{\equiv}\left\{y \in R^{m} ; y_{i}>0 ; \forall i=\overline{1, m}\right\}$ denotes a positive orthant of the space $\mathrm{R}^{\mathrm{m}}$, particularly, if $m=1$ we have $R_{++}^{1} \stackrel{\text { def }}{\equiv}(0,+\infty) ; R_{+}^{m} \stackrel{\text { def }}{\equiv}\left\{y \in R^{m} ; y_{i} \geq 0 ; \forall i=\overline{1, m}\right\}$ denotes a nonnegative orthant of the space $\mathrm{R}^{\mathrm{m}}$, in particular, if $m=1$ we have $R_{+}^{1} \stackrel{\text { def }}{\equiv}(0,+\infty) c_{i, i+j}^{k}(t):\left[T_{\text {start }}, T_{\text {end }}\right] \rightarrow R_{++}^{1}$ denotes the cost of transportation of an unit of the cargo loaded in $i-\operatorname{th}(i=\overline{1,(n-1)})$ seaport which has to be transported to $(i+j)$-th $(j=\overline{1,(n-i)})$ seaports by the $k-$ th $(k=\overline{1, K})$ ship: in this work we consider only the case, when for $\forall t \in\left[T_{\text {start }}, T_{\text {end }}\right]$ holds $c_{i, i+j}^{k}(t) \equiv c_{i, i+j}^{k}=$ const; $p_{i, i+j}^{k} \in[0,100]$ denotes the percent of the cargo which is being transported by the $k-$ th $(k=\overline{1, K})$ ship and which, firstly, was loaded in the $i$-th $(i=\overline{1,(n-1)})$ seaport and, secondly, after its transportation in $(i+j)$-th $(j=\overline{1,(n-i)})$ seaport "settles down"/unloads in this seaport: for each fixed $k \in\{1,2, \ldots, K\}$ the identity $\sum_{j=1}^{n-i} p_{i, i+j}^{k}=100$ must hold for each $i=\overline{1,(n-1)} ; \quad a_{i} \in R_{+}^{1} \stackrel{\text { def }}{\equiv}(0,+\infty)$ denotes the amount of accumulated in $i$-th $(i=\overline{1,(n-1)})$ seaport cargo which can be exported: $a_{i}$ does not contain the amount of cargo which was brought to $i$-th seaport from the seaports $\overline{1,(i-1)} ; \quad\left\{a_{i}\right\}_{i=1,(i-1)} \mid=n-1 ; \quad b_{i}^{k} \in R_{+}^{1}$ denotes the total amount of cargo which can be exported from $i$-th $\overline{1,(i-1)}$; seaport to $(i+1)$-th seaport by the $k$-th $(k=\overline{1, K})$ ship: as opposed to $a_{i}$, $b_{i}^{k}$ contains the cargo loaded to the ship $k$ in seaports $\overline{1,(i-1)} ; x_{i, i+1}^{k} \in R_{+}^{1}$ denotes the amount of loaded in $i$-th $(i=\overline{1,(n-1)})$ seaport cargo which has to be delivered by the $k$-th $(k=\overline{1, K})$ ship to $(i+1)$-th seaport; $\quad x \equiv\left\{x_{i, i+1}^{k} \in R_{+}^{1}\right\}_{i=\overline{1,(n-1)}}^{k=\overline{1, K}}$ is a full matrix with the size $K \times(n-1)$, called the plan of the transportation and containing all sought-for variables.

Thus, among the listed above parameters the desired are $\left\{x_{i, i+1}^{k} \in R_{+}^{1}\right\}_{i=1,(n-1)}^{k=\overline{1, K}}$, the amount of what, obviously, equals $K \cdot(n-1)$, but the other $\left[K \cdot\left(n^{2}-1\right)+n+3\right]$ parameters, included $T_{\text {start }}$ and $T_{\text {end }}$, are supposed to be given source data. The problem is in determination of a transportation plan $x$, such that, firstly, the amount of transported cargo is maximal (first criterion), secondly, the total expenses of the transportation are minimal (second criterion) and, besides, generally speaking, these two criteria can be not equivalent.

\section{Construction of mathematical model}

Using introduced in the previous section denotations and assumptions we will formulate the criteria for our problem. Obviously, the maximality criterion of the cargo transported by the $k$-th $(k=\overline{1, K})$ ship is the following function

$w_{1}^{k}(x) \stackrel{\text { def }}{n-1} \sum_{i=1}^{k} x_{i, i+1}^{k}, \forall k=\overline{1, K}$. 
Therefore, the maximality criterion for all cargo transported by $K$ ships is determined by the additive function $\sum_{k=1}^{K} w_{1}^{k}$, so we have

$$
\max \left\{w_{1}(x) \equiv \sum_{i=1}^{\operatorname{def}} \sum_{k=1}^{n-1} x_{i, i+1}^{k}\right\}
$$

For construction of the minimality criterion for the expenses of the transportation of the cargo transported by the $k$-th $(k=\overline{1, K})$ ship, it is necessary to calculate these expenses for every $i$-th $(i=\overline{1,(n-1)})$ seaport (Fig. 1): for the Seaport \#1: $\left(\sum_{j=2}^{n} c_{1, j}^{k}-10^{-2} \cdot \sum_{m=2}^{n-1} p_{1, m}^{k} \sum_{j=m+1}^{n} c_{1, j}^{k}\right) \cdot x_{1,2}^{k} ; \ldots$; for the Seaport \#(n-1): $c_{n-1, n}^{k} \cdot x_{n-1, n}^{k}$. Summing up these $(n-1)$ expressions, we get the minimality criterion for the expenses of the cargo transportation by the $k-$ th $(k=\overline{1, K})$ ship:

$$
\min \left\{w_{2}^{k}(\tilde{x}) \equiv \sum_{i=1}^{\operatorname{def}}\left(\sum_{j=i+1}^{n-1} c_{i, j}^{k}-10^{-2} \cdot \sum_{m=i+1}^{n-1} p_{i, m}^{k} \sum_{j=m+1}^{n} c_{i, j}^{k}\right) \cdot x_{i, i+1}^{k}\right\}, \forall k=\overline{1, K}
$$

Now we proceed to constructing of essential constraints of the problem. As the sum $\sum_{k=1}^{K} x_{i, i+1}^{k}$ is the amount of cargo which initially belonged to $i-\operatorname{th}(i=\overline{1,(n-1)})$ seaport (implying that this has never been transported to this seaport form another) and which has to be transported to $(i+1)$-th seaport by the use of all $K$ ships, we can formulate the following $(n-1)$ inequalities:

$$
\sum_{k=1}^{K} x_{i, i+1}^{k} \leq a_{i}, \forall i=\overline{1,(n-1)}
$$

Then, as the amount of cargo transported between the seaports $i$ and $(i+1)(i=\overline{1,(n-1)})$ by the $k$-th $(k=\overline{1, K})$ ship must not exceed the boat load $b_{i}^{k}$ of this ship, we can write: for transport route "Seaport $\# 1 \rightarrow$ Seaport \#2": $x_{1,2}^{k} \leq b_{1}^{k} ; \quad \ldots ;$ for transport route "Seaport $\#(\mathrm{n}-1) \rightarrow$ Seaport $\#(\mathrm{n})$ ": $\sum_{j=1}^{n-1}\left(1-10^{-2} \cdot \sum_{m=1}^{n-j-1} p_{j, j+m}^{k}\right) \cdot x_{j, j+1}^{k}+x_{n-1, n}^{k} \leq b_{n-1}^{k}$. Therefore, we have the following $K \cdot(i+1)$ inequalities:

$$
\sum_{j=1}^{i} x_{j, j+1}^{k}-10^{-2} \cdot \sum_{m=1}^{n-2} \sum_{j=1}^{i-m} p_{j, j+m}^{k} \cdot x_{j, j+1}^{k} \leq b_{i}^{k}, \forall(i=\overline{1,(n-1)} ; k=\overline{1, K})
$$

Finally, as $x_{i, i+1}^{k}$ denotes the amount of cargo, we can write the following sign constraints:

$$
x_{i, i+1}^{k} \geq 0 \quad \forall(i=\overline{1,(n-1)}, k=\overline{1, K})
$$

Thus, combining the formulas (1), (3)-(6), we get the following mathematical model of the considered problem: it is required to determine the values of the variables $\left\{x_{i, i+1}^{k}\right\}_{i=1, \overline{1,(n-1)}}^{k=\overline{1, K}}$ which satisfies $(2 \cdot K+1) \cdot(n-1)$ constraints 


$$
\left\{\begin{array}{l}
\sum_{k=1}^{K} x_{i, i+1}^{k} \leq a_{i}, \forall i=\overline{1,(n-1)}, \\
100 \cdot\left(\sum_{j=1}^{i} x_{j, j+1}^{k}-b_{i}^{k}\right)-\sum_{m=1}^{n-2} \sum_{j=1}^{i-m} p_{j, j+m}^{k} \cdot x_{j, j+1}^{k} \leq 0, \forall(i=\overline{1,(n-1)} ; k=\overline{1, K}), \\
x_{i, i+1}^{k} \geq 0, \forall(i=\overline{1,(n-1)}, k=\overline{1, K}),
\end{array}\right.
$$

maximize the criterion

$w_{1}(x) \stackrel{\text { def }}{\equiv} \sum_{i=1}^{n-1} \sum_{k=1}^{K} x_{i, i+1}^{k}$

and minimize the criteria

$$
w_{2}(x) \stackrel{\text { def }}{\equiv} \sum_{k=1}^{K} \sum_{i=1}^{n-1}\left(\sum_{j=i+1}^{n} c_{i, j}^{k}-10^{-2} \cdot \sum_{m=i+1}^{n-1} p_{i, m}^{k} \sum_{j=m+1}^{n} c_{i, j}^{k}\right) \cdot x_{i, i+1}^{k} .
$$

As the model (7)-(9) is a multicriteria problem, speaking about the solution of the model (6)-(8), we will imply its Pareto optimal solution, which represents (Tuy et al., 2008; Deb, 2001; Steuer, 1986; Coello Coello and Lamont, 2004) generalization of concept of numerical function's optimum point for the case, when we have many function, notably, the solution of a multicriteria problem is a Pareto optimal solution if the value of every criterion can be improved only at the cost of worsening the values of other criteria. The ultimate objective of this work consists in finding a transportation plan $X$ which is a Pareto optimal solution of the problem. We will shortly call this plan an effective plan.

\section{Solving the proposed models (6)-(8) and (2), (6), (7) on the assumption of equivalence of criteria}

In this section we will investigate introduced in the section 3 bi-criteria (6)-(8) and $(\mathrm{K}+1)$-criterion problems (2), (6), (7). First of all, we will try to introduce and explain a transformation which sets biunivocal correspondence between the three-index desired variables $x_{i, i+1}^{k}(k=\overline{1, K} ; i=\overline{1,(n-1)})$ and new one-index variables $y_{j}(j=\overline{1, K \cdot(n-1)})$, using which, because of many reasons (not only with the objective of simplification of the variables appearance), is rational to reformulate and investigate the models (7)-(9) and (2), (7), (8).

So, instead of the variables $x_{i, i+1}^{k}(k=\overline{1, K} ; i=\overline{1,(n-1)})$ we will introduce new variables $y_{j}(j=1, K \cdot(n-1))$, where index $j \in\{1,2, \ldots, K \cdot(n-1)\}$ of the variable $y_{j}$ and indexes $i, k(i \in\{1,2, \ldots, n-1\} ; k \in\{1,2, \ldots, K\})$ of the variable $x_{i, i+1}^{k}$ are connected by the following relations:

$j=(n-1) \cdot(k-1)+i$ for each fixed pair $i, k$;

$$
\left\{\begin{array}{l}
i \equiv j(\bmod (n-1)), \\
k=1+\frac{j-i}{n-1}
\end{array} \text { for each fixed } j\right.
$$

The role of the transformation (9) consists in the following: for each fixed pair of indexes, $i, k$ of the variable $x_{i, i+1}^{k}$ the transformation (9) puts in correspondence unique index $j$ of the variable $y_{j}$. In other words, the transformation (9) uniquely maps (uniqueness is obvious as index $j$ runs over the rows 
of the matrix $x)$ the three-index variable $x_{i, i+1}^{k}$ into one-index variable $y_{j}: x_{i, i+1}^{k} \stackrel{(10)}{\longrightarrow} y_{j}=y_{i+(k-1) \cdot(n-1)}$. Therefore, instead of the desired plan $x$ of transportation we obtain the same plan of transportation in the form of a column-vector $y \equiv\left(y_{1}, y_{2}, \ldots, y_{K \cdot(n-1)}\right)^{T}$. The transformation (140) is inverse transformation to the transformation (10). Notably, for each fixed index $j$ of the variable $y_{j}$ the transformation (10) puts in correspondence an unique pair of indexes $i, k$ of the variable $x_{i, i+1}^{k}$. Let's prove that the transformation (10), firstly, returns us positive integers $i, k$; and, secondly, unambiguously determines the pair of indexes $i, k$. First of all, let's prove the naturalness of the numbers $i, k$. Naturalness of $i$ immediately follows from the first formula of the transformation (10), which is nothing else than the definition of congruence $(n-1)$ between numbers $i$ and $j$ (Weil, 2013; Davenport, 2008). Now let's prove that $k \in N$ As $j \in N$ it is obvious, that there always exist numbers $m_{1} \in N \bigcup\{0\}, m_{2} \in N$, such that $j=(n-1) \cdot m_{1}+m_{2}$. Then, from $i \in\{1,2, \ldots, n-1\}$ and $i \equiv j(\bmod (n-1))$ follows that $i=m_{2}$, i.e. $j=(n-1) \cdot m_{1}+i$. As from the uniquely forward transformation (10) follows unique determination of the index $k$, we can write that $k=1+\frac{j-i}{n-1}=1+\frac{(n-1) \cdot m_{1}+i-i}{n-1}=1+m_{1}$. As $m_{1} \in N$, from the last expression follows $k=\left(1+m_{1}\right) \in N$. The proof of naturalness the pair of indexes $i, k$, determined by the transformation (10) is complete. Now we have to prove that the choice of natural indexes $i, k$ by (10) is unique. The proof of this statement will be based on the definition of the complete residue class modulo $m$ concept (Weil, 2013; Davenport, 2008): the set $A$ is called a complete residue class modulo $m$, if for $\forall l \in\{0,1,2, \ldots, m-1\}$ there exists an element $a \in A$, such that $l \equiv a(\bmod m)$. As for out matrix $x$ (i.e. the transportation plan) the column index $i$ varies from 1 to $(n-1)$ included, the set $\{1,2, \ldots, n-1\}$ is a complete residue class modulo $(n-1)$, i.e. for every two different elements of the set $\{1,2, \ldots, n-1\}$ the remainders of their division by $(n-1)$ are different too: $\forall i_{1}, \forall i_{2} \in\{1,2, \ldots, n-1\}: i_{1} \neq i_{2} \Rightarrow i_{1} \neq i_{2}(\bmod (n-1))$. In other words, we have proved the uniqueness of the index $i$. In view of the fact that with fixed values of $j$ and $n$ the desired index $k$, determined by the second formula of the transformation (10) is a single-valued function of an argument $i$ from the proven above fact about the uniqueness of the index $i$ follows the uniqueness of the index $k$. The proof of the inverse transformation (11) is complete. Thus, in future we will use new variables $\left\{y_{j}\right\}_{j=1, K \cdot(n-1)}$ instead of the old variables $\left\{x_{i, i+1}^{k}\right\}_{i=1,(n-1)}^{k=\overline{1, K}}$.

\subsection{Transforming the bi-criteria problem (6)-(8) into a simple linear programming problem}

Let's reformulate the model (6)-(8), using new variables $\left\{y_{j}\right\}_{j=1, K \cdot(n-1)}$, in which the index $j$ is determined in accordance with the transformation (9):

$$
\begin{aligned}
& \max \left\{w_{1}(y) \stackrel{\text { def }}{\equiv} \sum_{j=1}^{K \cdot(n-1)} y_{j}\right\}, \\
& \min \left\{w_{2}(y) \stackrel{\text { def }}{\equiv} \sum_{j=1}^{K \cdot(n-1)} \xi_{j} \cdot y_{j}\right\},
\end{aligned}
$$

subject to 


$$
\left\{\begin{array}{l}
\sum_{j=1}^{K} y_{i+(j-1) \cdot(n-1)} \leq a_{i}, \quad \forall i=\overline{1,(n-1)}, \\
\sum_{m=1}^{i-1} \pi_{i}^{k}(m) \cdot y_{m+(k-1) \cdot(n-1)}+y_{i+(k-1) \cdot(n-1)} \leq b_{i}^{k}, \forall(i=\overline{1,(n-1)} ; k=\overline{1, K}), \\
y_{j} \geq 0, \forall j=\overline{1, K \cdot(n-1)} .
\end{array}\right.
$$

For each value of $i \quad(i=\overline{2,(n-1)})$ and $k(k=\overline{1, K})$ the coefficients $\pi_{i}^{k}(m)$, which appear in the second line of the constraints system (14) are calculated using the following formula:

$$
\pi_{i}^{k}(m) \stackrel{\text { def }}{\equiv} 1-10^{-2} \cdot \sum_{j=m+1}^{i} p_{m, j}^{k}
$$

Thus, instead of initial bi-criteria model (6)-(8) we have obtained an equivalent bi-criteria problem (11)-(14), for which finding of the Pareto optimal solution will be achieved by using Gembicki goal attainment method (Gembicki, 1973; Gembicki and Haimes, 1975). We introduce the denotation

$$
Y_{(14)} \stackrel{\text { def }}{\equiv}\left\{y \in R_{+}^{1}: \sum_{j=1}^{K} y_{i+(j-1) \cdot(n-1)} \leq a_{i}, \forall i=\overline{1,(n-1)} ; \sum_{m=1}^{i-1} \pi_{i}^{k}(m) \cdot y_{i+(k-1) \cdot(n-1)} \leq b_{i}^{k}, \forall(i=\overline{1,(n-1)} ; k=\overline{1, K})\right\}
$$

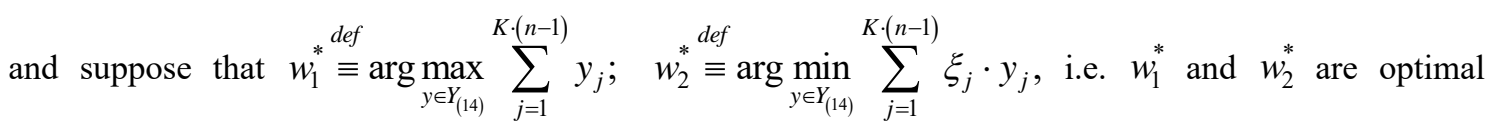
solutions of the composite one-criterion problems (11), (13), (14) and (12)-(14), respectively. Then, by virtue of the results of the previous sections, we can state that Pareto optimal solution of the problem (12)(16) (therefore, of the initial problem (6)-(8), due to one-to-one transformations (9), (10)) is the optimal solution of the following one-criterion problem of linear programming:

$$
\min \left\{M_{1} \cdot \sum_{i=1}^{(K+1) \cdot(n-1)+2} z_{i+(2 \cdot K+1) \cdot(n-1)+2}+z_{i+(3 \cdot K+2) \cdot(n-1)+5}-z_{i+(3 \cdot K+2) \cdot(n-1)+6}\right\},
$$

Subject to:

$$
\left\{\begin{array}{l}
\sum_{j=1}^{K} z_{i+(j-1) \cdot(n-1)}+z_{i+K \cdot(n-1)}+z_{i+(2 \cdot K+1) \cdot(n-1)+2}=a_{i}, \forall i=\overline{1,(n-1)}, \\
\sum_{m=1}^{i-1} \pi_{i}^{k}(m) \cdot z_{m+(k-1) \cdot(n-1)}+z_{i+(k-1) \cdot(n-1)}+z_{i+(K+k) \cdot(n-1)} \\
+z_{i+(2 \cdot K+k+1) \cdot(n-1)+2}=b_{i}^{k}, \forall(i=\overline{1,(n-1)} ; k=\overline{1, K}), \\
\sum_{j=1}^{K \cdot(n-1)} z_{j}-z_{(2 \cdot K+1) \cdot(n-1)+1}+z_{(3 \cdot K+2) \cdot(n-1)+3}+w_{1}^{*} \cdot z_{i+(3 \cdot K+2) \cdot(n-1)+5}-w_{1}^{*} \cdot z_{i+(3 \cdot K+2) \cdot(n-1)+6}=w_{1}^{*}, \\
K \cdot(n-1) \\
\sum_{j=1} \xi_{j} \cdot z_{j}+z_{(2 \cdot K+1) \cdot(n-1)+2}+z_{(3 \cdot K+2) \cdot(n-1)+4}-w_{2}^{*} \cdot z_{i+(3 \cdot K+2) \cdot(n-1)+5}+w_{2}^{*} \cdot z_{i+(3 \cdot K+2) \cdot(n-1)+6}=w_{2}^{*}, \\
z_{j} \geq 0, \forall j=\overline{1,((3 \cdot K+2) \cdot(n-1)+6),}
\end{array}\right.
$$


where $z \stackrel{\text { def }}{\equiv}\left\{z_{i} \in R_{+}^{1}\right\}_{i=1,((3 \cdot K+2) \cdot(n-1)+6)}$ is an introduced for the convenience column-vector, first $K \cdot(n-1)$ elements of what form the desired vector $y$ of the problem (11)-(14), i.e. $z_{i}=y_{i}$ for $\forall i=\overline{1, K \cdot(n-1)}$; as $M$ in (18) we can choose sufficiently great number, for example, the following number:

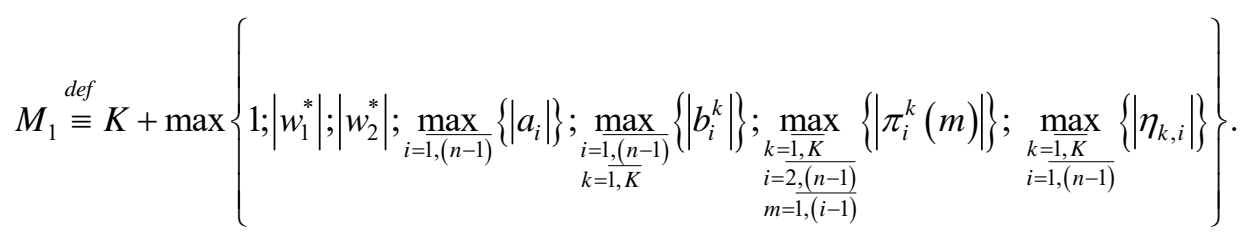

So, let the vector $z^{\text {opt }} \in R_{+}^{(3 \cdot K+2) \cdot(n-1)+6}$ be the solution of the problem (16)-(18), found but a decomposition algorithm or the parallelizing realization of the simplex method. Then, $y^{\text {Pareto }}=\left(z_{1}^{\text {opt. }}, \ldots, z_{K \cdot(n-1)}^{\text {opt. }}\right)$ is a trade-off solution of the bi-criteria problem (11)-(14). Therefore, by using the inverse transformation (10), we can uniquely determine Pareto optimal transportation plan $x^{\text {Pareto }} \in \square_{+}^{1}(K \times(n-1))$ :

$x^{\text {Pareto }}=\left(\begin{array}{cccc}z_{1}^{\text {opt. }} & z_{2}^{\text {opt. }} & \ldots & z_{n-1}^{\text {opt. }} \\ z_{n}^{\text {opt. }} & z_{n+1}^{\text {opt. }} & \ldots & z_{2 \cdot(n-1)}^{\text {opt. }} \\ \ldots & \ldots & \ldots & \ldots \\ z_{(K-1) \cdot(n-1)+1}^{\text {opt. }} & Z_{(K-1) \cdot(n-1)+2}^{\text {opt. }} & \ldots & z_{K \cdot(n-1)}^{\text {opt. }}\end{array}\right)$.

\subsection{Transforming the $(\mathrm{K}+1)$-criterion problem (2), (6), (7) into a simple linear programming problem}

By analogy with the subsection (3.1), we, firstly, reformulate the $(K+1)$-criterion problem (2), (6), (7) in new variables $\left\{y_{j}\right\}_{j=1, K \cdot(n-1)}$, in which the index $j$ is calculated in accordance with the transformation (9), and then reduce the obtained $(\mathrm{K}+1)$-criterion problem to a simple linear programming problem using the Big M Method once again.

Thus, in new variables we have the following $(\mathrm{K}+1)$-criterion problem:

$$
\begin{aligned}
& \max \left\{w_{1}(y) \stackrel{\operatorname{def}}{\equiv} \sum_{j=1}^{K \cdot(n-1)} y_{j}\right\}, \\
& \min \left\{w_{2}^{k}(\tilde{x}) \equiv \sum_{i=1}^{\operatorname{def}} \eta_{k, i} \cdot y_{i+(k-1) \cdot(n-1)}\right\}, \forall k=\overline{1, K},
\end{aligned}
$$

subject to

$$
\left\{\begin{array}{l}
\sum_{j=1}^{K} y_{i+(j-1) \cdot(n-1)} \leq a_{i}, \forall i=\overline{1,(n-1)}, \\
\sum_{m=1}^{i-1} \pi_{i}^{k}(m) \cdot y_{m+(k-1) \cdot(n-1)}+y_{i+(k-1) \cdot(n-1)} \leq b_{i}^{k}, \forall(i=\overline{1,(n-1)} ; k=\overline{1, K}), \\
y_{j} \geq 0, \forall j=\overline{1, K \cdot(n-1)}
\end{array}\right.
$$

where $\pi_{i}^{k}(m)$ and $\eta_{k, i}$ are calculated using, respectively, the formulas (14). 
As it was done in the previous subsection, we use the goal attainment method of Gembicki as well as the Big M Method, but now instead of the (K+1)-criterion problem (20)-(22) we will have the following one-criterion problem:

$$
\min \left\{M_{2} \cdot \sum_{i=1}^{(K+1) \cdot n} z_{i+K \cdot(2 \cdot n-1)}+Z_{(3 \cdot K+1) \cdot n-K+1}-z_{(3 \cdot K+1) \cdot n-K+2}\right\}
$$

subject to

$$
\left\{\begin{array}{l}
\sum_{j=1}^{K} z_{i+(j-1) \cdot(n-1)}+z_{i+K \cdot(n-1)}+z_{i+K \cdot(2 \cdot n-1)}=a_{i}, \forall i=\overline{1,(n-1)}, \\
\sum_{m=1}^{i-1} \pi_{i}^{k}(m) \cdot z_{m+(k-1) \cdot(n-1)}+z_{i+(k-1) \cdot(n-1)}+z_{i+(K+k) \cdot(n-1)} \\
+z_{i+(K+k) \cdot(n-1)+n \cdot K}=b_{i}^{k}, \forall(i=\overline{1,(n-1)} ; k=\overline{1, K}), \\
\sum_{j=1}^{K \cdot(n-1)} z_{j}-z_{(2 \cdot K+1) \cdot(n-1)+1}+z_{(3 \cdot K+1) \cdot n-2 \cdot K}+w_{1}^{*} \cdot z_{(3 \cdot K+1) \cdot n-K+1}-w_{1}^{*} \cdot z_{(3 \cdot K+1) \cdot n-K+2}=w_{1} \\
\sum_{i=1}^{n-1} \eta_{k, i} \cdot z_{i+(k-1) \cdot(n-1)}+z_{2 \cdot K \cdot(n-1)+n}+z_{k+(3 \cdot K+2) \cdot n-2 \cdot K}-w_{2}^{k^{*}} \cdot z_{(3 \cdot K+1) \cdot n-K+1} \\
+w_{2}^{k^{*}} \cdot z_{(3 \cdot K+1) \cdot n-K+2}=w_{2}^{k^{*}}, \forall k=\overline{1, K}, \\
z_{j} \geq 0, \forall j=\overline{1,((3 \cdot K+1) \cdot n-K+2),}
\end{array}\right.
$$

where $w_{1}^{*} \stackrel{\text { def }}{\equiv} \arg \max _{y \in Y_{(14)}} \sum_{j=1}^{K \cdot(n-1)} y_{j} ; \quad w_{2}^{k^{*}} \stackrel{\operatorname{def}}{\equiv} \arg \min _{y \in Y_{(14)}} \sum_{i=1}^{n-1} \eta_{k, i} \cdot y_{i+(k-1) \cdot(n-1)}, \forall k=\overline{1, K}$; the set $Y_{(14)}$ is determined by (15); the number $M$ is sufficiently great and can be considered to be, for example,

$$
M_{2} \stackrel{\text { def }}{\equiv} K+\max \left\{1 ;\left|w_{1}^{*}\right| ; \max _{k=1, K}\left|w_{2}^{k^{*}}\right| ; \max _{i=1,(n-1)}\left\{\left|a_{i}\right|\right\} ; \max _{\substack{i=1,(n-1) \\ k=1, K}}\left\{\left|b_{i}^{k}\right|\right\} ; \max _{\substack{k=1, K \\ i=2,(n-1) \\ m=1,(i-1)}}\left\{\left|\pi_{i}^{k}(m)\right|\right\} ; \max _{\substack{k=1, K \\ i=1,(n-1)}}\left\{\left|\eta_{k, i}\right|\right\}\right\} .
$$

Supposing that the vector $z^{o p t} \in R_{+}^{(3 \cdot K+1) \cdot n-K+2}$ is the solution of the problem (25)-(27), the desired compromise plan of transportation for the initial model (2), (6), (7) is the matrix $x^{\text {Pareto }} \in R_{+}^{1}(K \times(n-1))$ of the form (19).

\section{Well-posedness research and construction of the regularized solution}

As it is well known (Tikhonov and Arsenin, 1977; Courant, 1989), a mathematical problem which corresponds to physical or economic, etc. reality, has to satisfy the following basic requirements: the solution must exist; the solution should be uniquely determined; the solution should depend continuously on the data (requirement of stability). This requirement of "stability" is not only essential for meaningful problems which describe the real processes, but also for approximation methods. Any problem which satisfies our three requirements will be called a properly posed (or well-posed) problem in the sense of Hadamard. Problems that are not well-posed in the sense of Hadamard are termed ill-posed. If the problem is well-posed, then it stands a good chance of solution on a computer using a stable algorithm. If it is not well-posed, it needs to be re-formulated for numerical treatment. Typically this involves including additional assumptions, such as smoothness of solution, etc. (Tikhonov and Arsenin, 1977). 
Obviously, if the investigated problem is about bilk cargo, then some of the source data, for example, at least $\left\{a_{i}\right\}_{i=1,(n-1)}$ and $\left\{b_{i}^{k}\right\}_{i=1, \overline{1,(i-1)}}^{k=\overline{1, K}}$, are given approximate. Generally, it makes sense to suppose that costs $i=\overline{1,(n-1)}\left\{c_{i, i+j}^{k}\right\}_{j=1,(n-i)}^{k=\overline{1, K}}$ are given approximately too and that we know the order of approximation of the source data.

It is not difficult to see that one-criterion problems (16)-(18) and (23)-(25) can be rewritten in a more compact form:

$\left\{\begin{array}{l}\min _{z \in Z}\{L(z)\}, \\ Z \stackrel{\operatorname{def}}{\equiv}\left\{z \in R_{+}^{\beta}: A z=u ; A \in R^{1}(\alpha \times \beta), u \in R^{\alpha},(\alpha, \beta) \in N\right\} .\end{array}\right.$

In the case if the problem (18)-(20) is being investigated, then the objective function $L(z)$ has the from (18); the matrix $A$ contains the variables held constants in the left side of the system (19); the columnvector $u$ contains constants in the right side of the system $(19) ; \quad \alpha=(K+1) \cdot(n-1)+2$; $\beta=(3 \cdot K+2) \cdot(n-1)+6$. in the case if the problem (25)-(27) is being investigated, then the objective function $L(z)$ has the form (23); the matrix $A$ contains the variables held constants in the left-hand side of the system (24); the column-vector $u$ contains constants in the right side of the system (24); $\alpha=(K+1) \cdot n ; \beta=(3 \cdot K+1) \cdot n-K+2$.

Let's suppose that instead of the problem (26) with the precisely given data we have the following approximating problem with given approximately source data:

$$
\left\{\begin{array}{l}
\min _{z \in Z_{\delta}}\{L(z)\}, \\
Z_{\delta} \stackrel{\operatorname{def}}{\equiv}\left\{z \in R_{+}^{\beta}: A_{\delta} z=u_{\delta} ; A_{\delta} \in R^{1}(\alpha \times \beta), u_{\delta} \in R^{\alpha},(\alpha, \beta) \in N\right\} .
\end{array}\right.
$$

where in the capacity of the proximity measure between the corresponding elements (vectors, matrixes) is chosen the Euclidean metric:

$$
\left\|A-A_{\delta}\right\|=\sqrt{\sum_{i=1}^{\alpha} \sum_{j=1}^{\beta}\left(A_{i, j}-\left(A_{\delta}\right)_{i, j}\right)^{2}} \leq \delta ;\left\|u-u_{\delta}\right\|=\sqrt{\sum_{i=1}^{\alpha}\left(u_{i}-\left(u_{\delta}\right)_{i}\right)^{2}} \leq \delta .
$$

Obviously, because of source data precision $\left\{A_{\delta} ; u_{\delta}\right\}$, the request of the system $A z=u$ to be a linearly independent system is an unlawful request, so establishment of this fact is practically impossible. Besides, because of source data precision, the system $A_{\delta} z=u_{\delta}$ appears to be an inconsistent system. In other words, the first requirement for well-posedness in the sense of Hadamard is broken. Further, as it is shown in (Tikhonov et al., 1969) during the process of solving a particular practical problem (in this work a problem of finding optimal quarterly plans on multiproduct manufacturing was investigated) using the simplex method, is possible a situations, when for relatively close (with error less than 1\%) optimal values of the objective linear function $L(z)$ the amount of items planned to be manufactured in accordance to these optimal plans varies within the range of some hundreds, so the investigated problem appears to be an unstable problem, i.e. the third requirement for well-posedness in the sense of Hadamard is broken. Finally, if we suppose that all source data are given precisely and $Z \neq \varnothing$, then, as the following example (Tikhonov, 1966) shows, the problem (26) (all the more the approximating problem (27), (28)) can have not only one solution: minimum of the objective function $L(z)=z_{3}$ on the set $Z=\left\{z \in R_{+}^{3}: z_{1}-z_{2}=0\right\}$ is equal to zero and is reached in any point $z=\left(z_{1}, z_{1}, 0\right), \forall z_{1} \geq 0$, i.e. on the half-line determined by the equation $z_{2}=z_{1}, z_{3}=0$. In other words, the second requirement for well-posedness in the sense of Hadamard is broken, and, therefore, in cases like this it is required to impose additional condition on the desired solution. In case of the investigated problem being a problem of optimal planning (as the problem investigated in this work is), the additional condition, needed for ensuring of unambiguity, can be 
formulated in the following way: let a cargo transportation or any other work be performed according to the plan $z^{*}$ and let it be required to change this plan, because of change of the source data. Obviously, new source data correspond to new optimal plans. Thus, it is absolutely natural to choose the plan which is the least different from the original plan $z^{*}$, because this criterion of choice will entail the least costs of organizational reconstructions, if they were not considered in the formulation of the problem. As the measure of deviation of the new $z^{\text {new }}$ and the original $z^{*}$ plans can be accepted any positively definite quadric quantic $\sum_{i=1}^{\beta} \sum_{j=1}^{\beta} s_{i, j} \cdot\left(z_{i}^{\text {new }}-z_{i}^{*}\right) \cdot\left(z_{j}^{\text {new }}-z_{j}^{*}\right)$, particularly (for instance, if $s_{i, j}=0$ with $i \neq j$ ), can be accepted any weighted second-order deviation $\sum_{i=1}^{\beta} s_{i} \cdot\left(z_{i}^{\text {new }}-z_{i}^{*}\right)^{2}=\left\|z^{\text {new }}-z^{*}\right\|^{2}$. In other words, it is natural to demand for the plans $z^{\text {new }}$ and $z^{*}$ implementation of the inequality $\left\|z^{\text {new }}-z^{*}\right\|^{2} \leq\left\|z^{(28)}-z^{*}\right\|^{2}$, where vector $z^{(28)} \in R_{+}^{\beta}$ denotes any solution of the problem (28), i.e. $\forall z^{(39)} \in \operatorname{Arg} \min _{Z} L(z)$. For the problem (26) this vector $z^{\text {new }}$ is called a normal solution with respect to the original vector $Z^{*}$ (Tikhonov, 1966; Tikhonov et al., 1969; Tikhonov and Arsenin, 1977; Stefanov, 2001). In future we will use a shorter name "normal solution" and write it as $z^{\text {normal }}$. Obviously, in case of the problem (28) having an unique solution, it coincides with the normal solution $z^{\text {normal }}$. If the problem (28) has many solutions, then there obligatory exists its normal solution $z^{\text {normal }}$ : it follows from the fact that intersection of a finite number of closed sets is a closed set (Kolmogorov and Fomin, 1999), and the set on which the objective function $L(z)$ reaches its minimum is the intersection of three closed sets $\{z: A z=u\},\left\{z: z_{i} \geq 0, \forall i=\overline{1, \beta}\right\}$, $\left\{z: L(z)=L^{*}\right\}$, where $L^{*}$ is the lower bound of the values of $L(z)$ on the set $Z$. It is not difficult to see that the normal solution of the problem (26) is determined unambiguously (Tikhonov, 1966). Indeed, assuming that the problem (26) has two different normal solutions $z^{\text {normal(1) }}$ and $z^{\text {normal(2) }}$, we can assert that: any point $z=\sigma \cdot z^{\text {normal (1) }}+(1-\sigma) \cdot z^{\text {normal (2) }}, \sigma \in(0,1)$ of the segment $\left[z^{\text {normal(1) }}, z^{\text {normal(2) }}\right]$ satisfies the essential constraints as well as the sign constraints $z \geq 0$; objective function $L(z)$ reaches its maximum at all points of the segment $\left[z^{\text {normal(1) }}, z^{\text {normal (2) }}\right]$; the equality $\left\|z^{*}-z^{\text {normal (1) }}\right\|=\left\|z^{*}-z^{\text {normal (2) }}\right\| \quad$ holds, $\quad$ so $\quad$ it follows that $\left\langle z^{*}, z^{\text {normal (1) }}-z^{\text {normal (2) }}\right\rangle=\frac{\left\|z^{\text {normal (1) }}\right\|+\left\|z^{\text {normal (2) }}\right\|}{2} ;$ in the bisecting point of the segment $\left[z^{\text {normal (1) }}, z^{\text {normal (2) }}\right]$ we have $\left\|z^{\text {normal (1) }}-z^{*}\right\|>\left\|\frac{z^{\text {normal (1) }}+z^{\text {normal (2) }}}{2}-z^{*}\right\|$. Subject to $z^{\text {normal(1) }} \neq z^{\text {normal(2) }}$ the inequality contradicts the statement that for the problem (28) the vector (26) $Z^{\text {normal(1) }}$ is a normal solution with respect to the original vector $Z^{*}$. Thus, for a normal solution $Z^{\text {normal }}$ two first requirements for well-posedness in the sense of Hadamard are satisfied.

Summing up the above-mentioned, we can say that the problem (27), (28) with given approximately source data does not let us neither to judge whether the solution of the problem (26) with given precisely source data is stable, nor to judge whether it is unique even, if the problem (27), (28) has these properties. The exact solution of the problem (27), (28) with given approximately source data, as it was shown above, is inefficient for the investigation of the problem (26) with given precisely source data. From the point of view of available data, as the source data of the problem (26) can serve any source data set $\{A ; u\}$, satisfying the condition (28). We should note that, even if the problem (26) primordially was steady, adding any amount of linearly dependent equation to the essential constraints of the problem (26) makes it an unstable problem, though is stays equivalent to it in the classical sense. Therefore, it is necessary to develop such approach for solving problems of linear programming (particularly, the problems (26)-(30)) for which 
need no assumption about linear independence of the essential constraints. Below we shortly describe a stable method of finding approximate normal solution both for the problem (26)-(30). At first, this method was described in the fundamental article (Tikhonov, 1966).

Let's consider the problem (26), where the source data are given precisely. The point of this stable method of finding approximate normal solution of this problem consists in taking for an approximate solution the following element

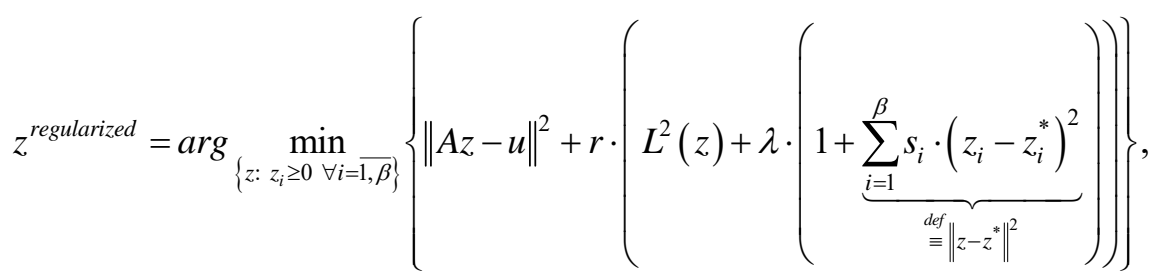

where the parameter $r \in R_{++}^{1}$ is called a regularization parameter; $\lambda \in R_{++}^{1}$ is a parameter; vector $z^{*}=\left\{z_{i}^{*}\right\}_{i=\overline{1, \beta}}$ is the fixed original vector, with respect to which the normal solution is searched.

As the parametric functional $M_{\lambda}^{r}[z ; A, u, L] \stackrel{\text { def }}{\equiv}\|A z-u\|^{2}+r \cdot\left(L^{2}(z)+\lambda \cdot\left(1+\left\|z-z^{*}\right\|\right)\right)$, called Tikhonov functional is a quadratic functional and holds $M_{\lambda}^{r}[z ; A, u, L] \stackrel{\|z\| \rightarrow \infty}{\longrightarrow} \infty$, unique existence of the element $z^{\text {regularized }}$ from (29) is obvious.

Now we consider the problem (27), (28), where the source data are given approximately. The point of the stable method of finding approximate normal solution consists in taking in the capacity of an approximate solution the

$$
z^{\text {regularized }}=\arg \min _{\left\{z: z_{i} \geq 0 \forall i=\overline{1, \beta}\right\}}\left\{\left\|A_{\delta} z-u_{\delta}\right\|^{2}+r \cdot\left(L^{2}(z)+\lambda \cdot(1+\underbrace{\sum_{i=1}^{\beta} s_{i} \cdot\left(z_{i}-z_{i}^{*}\right)^{2}}_{\underset{\text { def }}{\equiv\left\|z_{z-z^{*}}\right\|^{2}}})\right\},\right.
$$

where $\lambda \in R_{++}^{1}$ is a parameter; vector $z^{*}=\left\{z_{i}^{*}\right\}_{i=\overline{1, \beta}}$ is the fixed original vector with respect to which the normal solution is sought; as the regularization parameter $r \in R_{++}^{1}$ can be taken a positive root of the equation

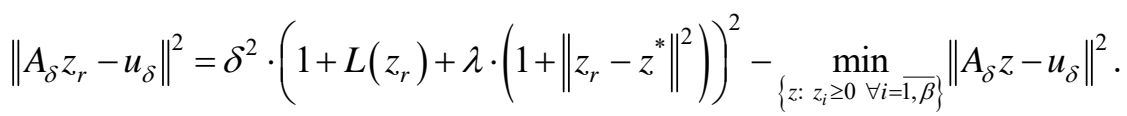

The equation (30) is called a generalized residual and the method of finding the regularization parameter $r \in R_{++}^{1}$ as a root of the equation (30) is called generalized residual principle and, at first, was described in the works (Goncharsky et al., 1973).

\section{Conclusions}

In the considered work, there is the mathematical model of optimal planning of multiproduct cargo transportation performed by ships through the given sea route, where every intermediate seaport is simultaneously a departure and destination point and in every of these intermediate seaports are performed handling operations, built. The built in this work mathematical model, being a multicriteria problem of linear programming, using the goal attainment method of Gembicki, is reduced to a scalar problem. Finally in this work is considered and substantiated a stable algorithm (on the basis of Tikhonov regularization) for finding an approximate solution of the derived scalar problem: there is shown applicability of this algorithm in case of given precisely source data as well as in case, when the source data are given approximately. 


\section{Acknowledgements}

For the last co-author the present article was executed within the framework of The State Research Programme "Next generation Information and Communication Technologies (NexIT)", Project No. 4.

\section{References}

1. Coello Coello, C.A., and Lamont, G.B. (2004) Applications of Multi-Objective Evolutionary Algorithms. Vol. 1: Advances in Natural Computation. New Jersey-London-Singapore-BerlinShanghai-Hong Kong-Taipei-Chennai: World Scientific, xxvii+761 p.

2. Courant, R. (1989) Partial Differential Equations. New York-London: Wiley VCH, xxii+830 p.

3. Davenport, H. (2008) The Higher Arithmetic: An Introduction to the Theory of Numbers. Cambridge, UK: Cambridge University Press, ix +239.

4. Deb, K. (2001) Multi-objective Optimization Using Evolutionary Algorithms. Chichester-New YorkWeinheim-Brisbane-Singapore-Toronto: John Wiley \& Sons, xix+497 p.

5. Gembicki, F.W. (1973) Vector Optimization for Control with Performance and Parameter Sensitivity Indices. Ph.D. Thesis, Department of System Engineering, Case Western Reserve University, Cleveland, USA, 204 p.

6. Gembicki, F.W., and Haimes, Y.Y. (1975) Approach to Performance and Sensitivity Multiobjective Optimization: The Goal Attainment Method. IEEE Transactions on Automatic Control, 29(6), pp. 769771.

7. Goncharsky, A.V., Leonov, A.S., and Yagola, A.G. (1973) A generalized residual principle. Computational Mathematics and Mathematical Physics, 13(2), pp. 294-302.

8. Kang, M.H., Choi, H.R., Kim, H.S., and Park, B.J. (2012) Development of a maritime transportation planning support system for car carriers based on genetic algorithm. Applied Intelligence, 36(3), pp. 585-604.

9. Liotta, G., Stecca, G., and Kaihara, T. (2015) Optimisation of freight flows and sourcing in sustainable production and transportation networks. International Journal of Production Economics, 164, pp. 351365.

10. Masane-Ose, J. (2014) Competitive position of the Baltic States Ports. Riga, Latvia: KPMG International Cooperative. (Transport \& Logistics, pp. 1-12; https://www.kpmg.lv)

11. Medvedeva, A.A. (2014) Opportunities to reduce aggregate expenditures by means of creating a strategic alliance by maritime cargo transportation. M.Sc. Thesis in Transport and Logistics. Riga, Latvia: Transport and Telecommunication Institute, Faculty of Transport and Logistics, 62 p.

12. Nikolaeva, L.L., and Tsymbal, N.N. (2005) Maritime Transportation. Odessa, Ukraine: FENIX Press, $424 \mathrm{p}$.

13. Song, D.-W., and Panayides, Ph.M. (2012) Maritime Logistics: A Complete Guide to Effective Shipping and Port Management. London-Philadelphia-New Delhi: Kogan Page, $344 \mathrm{p}$.

14. Steuer, R.E. (1986) Multiple Criteria Optimization: Theory, Computation, and Application. New York, USA: John Wiley \& Sons, $\mathrm{xx}+546 \mathrm{p}$.

15. Swiss Re Economic Research \& Consulting. (2001-2015) World Insurance Reports No 6/2001; No 6/2002; No 8/2003; No 3/2004; No 2/2005; No 5/2006; No 4/2007; No 3/2008; No 3/2009; No 2/2010; No 2/2011; No 3/2012; No 3/2013; No 3/2014; No 4/2015. Zurich, Switzerland: Swiss Re, sigma. http://www.swissre.com/sigma/

16. Tikhonov, A.N. (1966) Ill-posed optimal planning problems. Journal of Computational Mathematics and Mathematical Physics, 6(1), pp. 81-89.

17. Tikhonov, A.N., Karmanov, V.G., and Rudneva, T.L. (1969) On the stability of linear programming problems. In: Numerical Methods and Programming, XII. Moscow: Lomonosov Moscow State University Press, pp. 3-9.

18. Tikhonov, A.N., and Arsenin, V.Y. (1977) Solutions of Ill-Posed Problems. New York, USA: Halsted Press, $\mathrm{xiii}+258 \mathrm{p}$.

19. Tuy, H., Chinchuluun, A., Pardalos, P.M., Migdalas, A., and Pitsoulis, L. (2008) Pareto Optimality, Game Theory and Equilibria. New York: Springer, 871 p.

20. Wakeman, Th., and Bomba, M. (2010) Maritime Freight Transportation, National Economic Recovery, and Global Sustainability: Coordinating a Strategic Plan. Transportation Research Board of the National Academies of Sciences, Engineering, Medicine. TR News: Globalization and Transportation, 269, pp. 14-20.

21. Weil, A. (2013) Basic Number Theory. Berlin-Heidelberg: Springer-Verlag, xviii +316 p. 